\title{
Influence of the state of light on the optically induced interparticle interaction
}

\author{
Justo Rodríguez and David L. Andrews \\ School of Chemical Sciences, University of East Anglia, Norwich, NR4 7TJ, United Kingdom \\ (Received 13 November 2008; published 5 February 2009; corrected 24 February 2009)
}

\begin{abstract}
A general expression for the energy of interparticle interaction induced by an arbitrary mode of light is determined using quantum electrodynamics, and it is shown that the Casimir-Polder potential is included within this quantum result. Equations are also derived for the corresponding coupling induced by multimode number states of light, and the dependence of the pair energy on the Poynting vector and polarization state is determined. Attention is then focused on the interactions between particles trapped in counterpropagating coherent beams, both with and without interference, and it is shown that the results afford insights into the multiparticle structures that can be optically fabricated with counterpropagating input. Brief consideration is also given to the effect of squeezing the optical coherent state. Extending previous studies of optical binding in Laguerre-Gaussian beams, results are given for the case of particles trapped at radially different locations within the beam structure. Finally, consideration is given to interparticle interactions induced by broadband light, and it is shown how the length of optically fabricated particle chains can be controlled by the use of wavelength filters.
\end{abstract}

DOI: 10.1103/PhysRevA.79.022106

PACS number(s): 31.30.jh, 42.50.-p, 34.20.Gj, 85.85.+j

\section{INTRODUCTION}

Electromagnetic fields induce interparticle forces by generating static and dynamic polarizations. The best-known example of such interactions is the Casimir-Polder potential, which can be considered the consequence of an electric polarization induced in the interacting particles by vacuum field fluctuations [1,2]. Another, less familiar, example of such phenomena, also predicted using quantum electrodynamics (QED) [3], is an energy shift generated as a consequence of the polarization induced in particles by coherent states of light. The latter phenomenon, first experimentally observed by trapping particle pairs in optical tweezers [4] and now usually known as "optical binding," is currently the focus of a range of experimental and theoretical investigations [5-30]. The nature of the optical state in an optical trap is important not only for the organization of particles by gradient forces, but also to expedite determination of the form of optically induced interparticle interactions. Laboratory observations are often, for instance, effected in the standing waves of counterpropagating beams, in order to circumvent any effects due to optical pressure; particles trapped by gradient forces are then organized within the maxima of irradiance by optical binding forces $[12,20]$. Another strategy that is employed is to increase the coherence length of the beams, to avoid the interference between the counterpropagating optical fields; consequently optical binding is observed to play a more important role in particle organization [15]. It has also been shown that particles trapped in the intensity rings of Laguerre-Gaussian light can be organized within these rings by optical binding forces [21]. Particularly relevant to our present study, a recently developed optical trap fabricated using a white light (supercontinuum) source has permitted interactions to be studied without interference between the particle backscatter field and the beam field, such that it proves possible for particles to be entirely organized along the propagation direction of these beams by the optical binding forces $[19,30]$. Recognizing the importance of such fac- tors, it is timely to undertake a detailed analysis of the effects of these and other optical states on the optically induced interparticle interaction; this is the subject of the paper that follows.

First, in Sec. II, an expression for the interparticle interaction induced by an arbitrary single mode of monochromatic light is determined using QED, and its relationship with the Casimir-Polder potential is established. Next, in Sec. III, equations for the interaction induced by multimode number states of light are derived for various Poynting vector and polarization conditions. The characteristics of the interaction in coherent counterpropagating beams are analyzed in Sec. IV, and the effect on the interaction of squeezing the coherent state is briefly discussed. In Sec. V, we extend previous QED studies of interparticle interaction in LaguerreGaussian beams, to consider particles trapped in different radial locations of the beam. Lastly, in Sec. VI, an analytical result is derived for the form of an optical binding interaction induced by broadband light, and it is shown how the length of optically fabricated particle chains can be controlled by judicious filtering of the optical input.

\section{INTERPARTICLE INTERACTION INDUCED BY A SINGLE MODE OF LIGHT}

Here, a QED representation cast in terms of induced moments [31] is used to derive a general expression for the interparticle potential energy induced by an arbitrarily offresonant mode of light with a discrete wavelength. The results derived with this method in this and the following sections apply specifically to Rayleigh particles, but they can be extended to larger particles by summing over the optical response of small domains, at different locations within each particle $[26,27]$. To begin, the electrical polarization of a particle, $\xi$, with position vector $\mathbf{r}^{(\xi)}$ and electric susceptibility $\boldsymbol{\chi}^{(\xi)}$, subject to a transverse electric displacement whose field operator is $\mathbf{d}^{\perp}\left(\mathbf{r}^{(\xi)}\right)$, is written as 


$$
P_{i}^{\mathrm{ind}}(\xi)=\chi_{i j}^{(\xi)} d_{j}^{\perp}\left(\mathbf{r}^{(\xi)}\right),
$$

where the convention of implied summation over repeated indices is used; here also, the operator $\mathbf{d}^{\perp}\left(\mathbf{r}^{(\xi)}\right)$ is given by the following mode expansion:

$$
\begin{aligned}
\mathbf{d}^{\perp}\left(\mathbf{r}^{(\xi)}\right)= & -i \sum_{\mathbf{p}, \varepsilon}\left(\frac{\hbar c p \varepsilon_{0}}{2 V}\right)^{1 / 2} \\
& \times\left[\mathbf{e}^{(\varepsilon)}(\mathbf{p}) a^{(\varepsilon)}(\mathbf{p}) e^{i \mathbf{p} \cdot \mathbf{r}^{(\xi)}}-\overline{\mathbf{e}}^{(\varepsilon)}(\mathbf{p}) a^{\dagger(\varepsilon)}(\mathbf{p}) e^{i \mathbf{p} \cdot \mathbf{r}^{(\xi)}}\right],
\end{aligned}
$$

where $a^{(\varepsilon)}(\mathbf{p})$ and $a^{\dagger(\varepsilon)}(\mathbf{p})$ denote the photon annihilation and creation operators, respectively, for the radiation mode $(\mathbf{p}, \varepsilon) ; \mathbf{e}^{(\varepsilon)}(\mathbf{p})$ is the corresponding polarization vector, and $\overline{\mathbf{e}}^{(\varepsilon)}(\mathbf{p})$ is its conjugate; $V$ is an arbitrary quantization volume. The energy operator, for an interaction between particles $A$ and $B$ separated by a displacement vector $\mathbf{R}$, is given by

$$
\Delta E=\operatorname{Re}\left[P_{i}^{\text {ind }}(A) V_{i j}(k, \mathbf{R}) \bar{P}_{j}^{\text {ind }}(B)\right],
$$

featuring a retarded interaction tensor whose explicit form is as follows:

$$
\begin{aligned}
V_{i j}(k, \mathbf{R})= & \frac{e^{i k R}}{4 \pi \varepsilon_{0} R^{3}}\left[(1-i k R)\left(1-3 \frac{R_{i} R_{j}}{R^{2}}\right)\right. \\
& \left.-(k R)^{2}\left(1-\frac{R_{i} R_{j}}{R^{2}}\right)\right] .
\end{aligned}
$$

The time-averaged expectation value (av) of the energy in Eq. (3) for an arbitrary optical input mode $|X\rangle$, of wave vector $\mathbf{k}$ and polarization $\lambda$, is

$$
\begin{aligned}
\langle\Delta E\rangle_{\mathrm{av}}= & \frac{\hbar c^{2} k}{V} \frac{\varepsilon_{0} \chi_{i k}^{(A)} \chi_{j l}^{(B)}}{c} \operatorname{Re}\left\{V_{i j}(k, \mathbf{R})\right. \\
& \times\left[\left\langle X\left|a^{(\lambda)}(\mathbf{k}) a^{(\lambda) \dagger}(\mathbf{k})\right| X\right\rangle e_{k}(\mathbf{k}, \lambda) \bar{e}_{l}(\mathbf{k}, \lambda) e^{-i \mathbf{k} \cdot\left(\mathbf{R}_{B}-\mathbf{R}_{A}\right)}\right. \\
& \left.\left.+\left\langle X\left|a^{(\lambda) \dagger}(\mathbf{k}) a^{(\lambda)}(\mathbf{k})\right| X\right\rangle \bar{e}_{k}(\mathbf{k}, \lambda) e_{l}(\mathbf{k}, \lambda) e^{i \mathbf{k} \cdot\left(\mathbf{R}_{B}-\mathbf{R}_{A}\right)}\right]\right\} .
\end{aligned}
$$

Using the commutation rule $a^{(\lambda)}(\mathbf{k}) a^{(\lambda) \dagger}(\mathbf{k})=a^{(\lambda) \dagger}(\mathbf{k}) a^{(\lambda)}(\mathbf{k})$ +1 , and expressing the energy shift in terms of the average mode occupation number $\left\langle X\left|a^{(\lambda) \dagger}(\mathbf{k}) a^{(\lambda)}(\mathbf{k})\right| X\right\rangle=\langle n\rangle_{\mathrm{av}}$, within the volume $V$, we write

$$
\begin{aligned}
\langle\Delta E\rangle_{\mathrm{av}}= & \frac{\hbar c^{2} k\langle n\rangle_{\mathrm{av}}}{V} \frac{\varepsilon_{0} \chi_{i k}^{(A)} \chi_{j l}^{(B)}}{c} \operatorname{Re}\left\{V_{i j}(k, \mathbf{R})\right\} \\
& \times\left[e_{k}(\mathbf{k}, \lambda) \bar{e}_{l}(\mathbf{k}, \lambda) e^{-i \mathbf{k} \cdot \mathbf{R}}+\bar{e}_{k}(\mathbf{k}, \lambda) e_{l}(\mathbf{k}, \lambda) e^{i \mathbf{k} \cdot \mathbf{R}}\right] \\
& +\frac{\hbar c^{2} k}{V} \frac{\varepsilon_{0} \chi_{i k}^{(A)} \chi_{j l}^{(B)}}{c} \operatorname{Re}\left[e_{i}(\mathbf{k}, \lambda) \bar{e}_{j}(\mathbf{k}, \lambda) e^{-i \mathbf{k} \cdot \mathbf{R}}\right],
\end{aligned}
$$

where $\hbar c^{2} k\langle n\rangle_{\mathrm{av}} / V$ is the mean irradiance of the input. The first term in (6), proportional to this irradiance, is the optically induced interparticle potential for any type of singlemode optical state. The second term is independent of a mode occupation number; on summing over Poynting vectors it signifies the Casimir-Polder potential [1].
When multimode states are to be considered, it is necessary to effect a summation over the salient modes, where necessary taking into account the interference between these modes to determine particle organization. Such interference generates gradient forces which are generally much larger than the optical binding forces. The former forces are, for instance, generally important for the organization of particles in counterpropagating beams $[26,27]$. The degree of interference in multimode states is limited by the uncertainty in phase, $\Delta \phi$, of each constituent mode-where the phase is $\phi=\mathbf{k} \cdot \mathbf{R}-c k t+\theta$, and $\theta$ is defined through $a^{(\lambda)}(\mathbf{k})|X\rangle$ $=|X| \exp (i \theta)|X\rangle$. For each occupied mode $|X\rangle$, the phase uncertainty is related to the corresponding uncertainty in the occupation number $\Delta n$ through the relation $\Delta n \Delta \cos \phi /\langle\sin \phi\rangle \geqslant 1 / 2 \quad$ [32]. An optical coherent state, which is a state of minimum uncertainty, satisfies the equality in the previous relation, and a superposition of several such laser fields generates interference. In contrast, the superposition of number states cannot generate interference, and in consequence optical binding alone determines particle organization. The detail of the interparticle interaction in multimode number states is considered in the following section.

\section{INTERPARTICLE INTERACTION INDUCED BY MULTIMODE NUMBER STATES OF LIGHT}

We now consider the interparticle interactions induced by high-repetition-rate pulses of multimode light, e.g., highpower solid-state Nd:glass lasers [33]. The state vector for the radiation can be written in terms of multimode number states of the general form $\left|n_{1,2 \ldots m}\right\rangle=\left|n_{1}\right\rangle\left|n_{2}\right\rangle \cdots\left|n_{m}\right\rangle$, where $\left|n_{1}\right\rangle,\left|n_{2}\right\rangle, \ldots$, and $\left|n_{m}\right\rangle$ are the kets associated with each mode and the time dependence of each mode occupancy is left implicit. Since $\left\langle n_{1,2 \ldots . m}\left|a^{\dagger}\left(n_{p}\right) a\left(n_{q}\right)\right| n_{1,2 \ldots m}\right\rangle=n_{p} \delta_{p q}$, we can treat the interaction of each mode with the particles individually $[34,35]$. To begin, we consider the condition in which the polarizations of all modes are identical and the beam axes collinear (both in copropagating and counterpropagating cases). In any such case, each mode generates a potential of the same form, and the energy shift is identical to that derived in Eq. (6) with the term $\hbar c^{2} k\langle n\rangle / V$ replaced by the irradiance of the beam.

Another configuration that can be realized is where a condition of random orientations applies to the Poynting vectors. To address such a case, it is first helpful to perform an average over laser polarizations. The energy shift that results from summing over the polarization of all laser modes can be expressed as

$$
\langle\Delta E\rangle=\frac{I \varepsilon_{0} \chi_{i k}^{(A)} \chi_{j l}^{(B)}}{c}\left(\delta_{i j}-\hat{k}_{i} \hat{k}_{j}\right) \operatorname{Re}\left[V_{k l}(k, \mathbf{R})\right] \cos (\mathbf{k} \cdot \mathbf{R})
$$

where $I$ is the irradiance of the nonpolarized beam. For randomly oriented Poynting vectors, we then need to write a sum over all possible wave vectors as a continuum integral [32]: 


$$
\sum_{\mathbf{k}}(\cdots) \Rightarrow \int(\cdots) k^{2} d k d \Omega \frac{V}{(2 \pi)^{3}} .
$$

The integration over the solid angle $\Omega$ can then be solved by using in Eq. (7) the following relation:

$$
\begin{aligned}
\frac{1}{4 \pi} \int\left(\delta_{i j}-k_{i} k_{j}\right) e^{-i \mathbf{k} \cdot \mathbf{R}} d \Omega & \\
= & \left(\delta_{i j}-\hat{R}_{i} \hat{R}_{j}\right) \frac{\sin (k R)}{k R}+\left(\delta_{i j}-\hat{R}_{i} \hat{R}_{j}\right)\left(\frac{\cos (k R)}{k^{2} R^{2}}-\frac{\sin (k R)}{k^{3} R^{3}}\right)
\end{aligned}
$$

the interparticle interaction energy then emerges as

$$
\begin{aligned}
\langle\Delta E\rangle= & -\frac{\hbar c}{4 \pi R^{3}} \int\left[\frac{\langle n(k)\rangle \hbar c^{2} k^{3}}{(2 \pi)^{3}}\right] \chi_{i k}^{(A)} \chi_{j l}^{(B)} \\
& \times\left(k R \sin (2 k R)+2 \cos (2 k R)-5 \frac{\sin (2 k R)}{k R}\right. \\
& \left.-\frac{6 \cos (2 k R)}{k^{2} R^{2}}+\frac{3 \sin (2 k R)}{k^{3} R^{3}}\right) d k,
\end{aligned}
$$

where the term in square brackets is the spectral irradiance of the light per unit solid angle in the wave-vector interval $(k, k+d k)$. The above expression has striking similarities to the Casimir-Polder potential [1] associated with vacuum field fluctuations; the integrand of (10) differs only from the integrand in Casimir-Polder result by the factor $2\langle n(k)\rangle$. We now turn to the interaction induced by coherent counterpropagating fields.

\section{INTERPARTICLE INTERACTION INDUCED BY COHERENT COUNTERPROPAGATING FIELDS}

Let us now consider the interparticle interaction induced by coherent counterpropagating fields. For the system considered in this section, the directions of the polarization vector and the propagation direction of the optical fields irradiating the particles define the $x$ and $z$ axes, respectively (i.e., one beam has its Poynting vector along the direction of the $z$ axis, $\mathbf{k}$, and the other one in the opposite direction, $-\mathbf{k}$; both beams have their polarization along the $x$ axis). In this coordinate system, we first determine the local rotationally averaged electric polarization of a molecule, $\xi$. When this molecule is subjected to the transverse electric displacement field operators $d_{x}^{\perp{ }^{(+)}}\left(r_{z}^{(\xi)}\right)$ and $d_{x}^{\perp(-)}\left(r_{z}^{(\xi)}\right)$ (the sign in the superscripts designating co- or counterpropagation with respect to $\mathbf{k})$, the mean electric polarization is first written as

$$
P_{x}^{\text {ind }}(\xi)=\chi^{(\xi)}\left[d_{x}^{\perp(+)}\left(r_{z}^{(\xi)}\right)+d_{x}^{\perp(-)}\left(r_{z}^{(\xi)}\right)\right],
$$

where $\chi^{(\xi)}$ is the isotropic electric susceptibility; and $d_{x}^{\perp( \pm)}\left(r_{z}^{(\xi)}\right)$ is given by

$$
\begin{aligned}
d_{x}^{\perp( \pm)}\left(r_{z}^{(\xi)}\right)= & -i\left(\frac{\hbar c k \varepsilon_{0}}{2 V}\right)^{1 / 2} \\
& \times\left[a( \pm \mathbf{k}) e^{i k\left( \pm r_{z}^{(\xi)}-c t\right)}-a^{\dagger}( \pm \mathbf{k}) e^{i k\left(\mp r_{z}^{(\xi)}+c t\right)}\right],
\end{aligned}
$$

where $a( \pm \mathbf{k})$ and $a^{\dagger}( \pm \mathbf{k})$ denote the photon annihilation and creation operators for the radiation modes $\mathbf{k}$ and $-\mathbf{k}$, respectively (we omit the polarization in the notation of these operators, since they can be distinguished by the Poynting vector). Also in (12), $V$ is an arbitrary quantization volume; the scalar $r_{z}^{(\xi)}$ is the projection on the $z$ axis of the position of the particle $\xi$, and $k$ is the modulus of the wave vector. The time dependence plays an important role in the calculations effected in this section, and we consider this role can be illustrated most clearly when the time dependence is explicit in the displacement operator; consequently, in Eq. (12) the unitary transformation $\hat{U}=\exp \left\{i\left[a^{\dagger}( \pm \mathbf{k}) a( \pm \mathbf{k})+1 / 2\right] k c t\right\}$ has been used to transfer the time dependence from the states of the radiation to the operators [34].

The expectation value of the energy shift, given by Eq. (3), can be determined by introducing the coherent state $\left|\alpha^{(+)}\right\rangle\left|\alpha^{(-)}\right\rangle$to describe the state of the radiation field, where $\left|\alpha^{(+)}\right\rangle$represents the state of the mode $\mathbf{k}$ and $\left|\alpha^{(-)}\right\rangle$the state of the mode $-\mathbf{k}$. The expectation value of the interparticle interaction potential energy, if there is a slight shift $\pm \Delta k$ between the modulus of the wave vector of the counterpropagating beams, can be expressed as follows;

$$
\begin{aligned}
\langle\Delta E\rangle= & \chi(k,-k)^{2} V_{x x}\left(k,\left(\mathbf{r}^{(A)}-\mathbf{r}^{(B)}\right)\right)\left(\frac{\hbar c k \varepsilon_{0}}{V}\right) \\
& \times\left\{\left\langle\alpha^{(+)}\left|a^{\dagger}(\mathbf{k}) a(\mathbf{k})\right| \alpha^{(+)}\right\rangle \cos \left[k\left(r_{z}^{(A)}-r_{z}^{(B)}\right)\right]\right. \\
& +\left\langle\alpha^{(-)}\left|a^{\dagger}(-\mathbf{k}) a(-\mathbf{k})\right| \alpha^{(-)}\right\rangle \cos \left[k\left(r_{z}^{(A)}-r_{z}^{(B)}\right)\right] \\
& +\frac{1}{2}\left[\left\langle\alpha^{(-)}\left|a^{\dagger}(-\mathbf{k})\right| \alpha^{(-)}\right\rangle\left\langle\alpha^{(+)}|a(\mathbf{k})| \alpha^{(+)}\right\rangle\right. \\
& \left.+\left\langle\alpha^{(-)}|a(-\mathbf{k})| \alpha^{(-)}\right\rangle\left\langle\alpha^{(+)}\left|a^{\dagger}(\mathbf{k})\right| \alpha^{(+)}\right\rangle\right] \\
& \left.\times \cos \left[k\left(r_{z}^{(A)}+r_{z}^{(B)}\right) \pm \Delta k t\right]\right\}
\end{aligned}
$$

where the terms involving a product of two annihilation or two creation operators have been discarded since they are rapidly oscillating and their time average is zero. This interparticle interaction potential, which has previously been determined for a field described by number states [31], is evaluated here using the commutation relation $a( \pm \mathbf{k}) a^{\dagger}( \pm \mathbf{k})=a^{\dagger}( \pm \mathbf{k}) a( \pm \mathbf{k})+1$, and the following eigenvalue equations for the coherent states involved: $a(\mathbf{k})\left|\alpha^{(+)}\right\rangle=\alpha^{(+)}\left|\alpha^{(+)}\right\rangle, \quad a(-\mathbf{k})\left|\alpha^{(-)}\right\rangle=\alpha^{(-)}\left|\alpha^{(-)}\right\rangle, \quad\left\langle\alpha^{(+)}\right| a^{\dagger}(\mathbf{k})$ $=\left\langle\alpha^{(+)}\right| \bar{\alpha}^{(+)}, \quad\left\langle\alpha^{(-)}\right| a^{\dagger}(-\mathbf{k})=\left\langle\alpha^{(-)}\right| \bar{\alpha}^{(-)}$. The positive and negative propagation components of the classical fields for the mode $\mathbf{k}$, for instance, correspond to $i\left(\hbar c k \varepsilon_{0} / 2 V\right)^{1 / 2} \alpha^{(+)} e^{i k\left(+r_{z}-c t\right)}$ and $-i\left(\hbar c k \varepsilon_{0} / 2 V\right)^{1 / 2} \bar{\alpha}^{(+)} e^{i k\left(-r_{z}+c t\right)}$, respectively $[32,36]$. The last term in Eq. (13), with the factor $\cos \left[k\left(r_{z}^{(A)}+r_{z}^{(B)}\right) \pm \Delta k t\right]$, is only present when $\Delta k=0$; otherwise its time average is zero. It is worth noting that, if the radiation field is described by number states this term again disappears; i.e., a degree of coherence is necessary for the simultaneous interaction with the two modes not to vanish.

The interparticle interaction can finally be written as follows: 

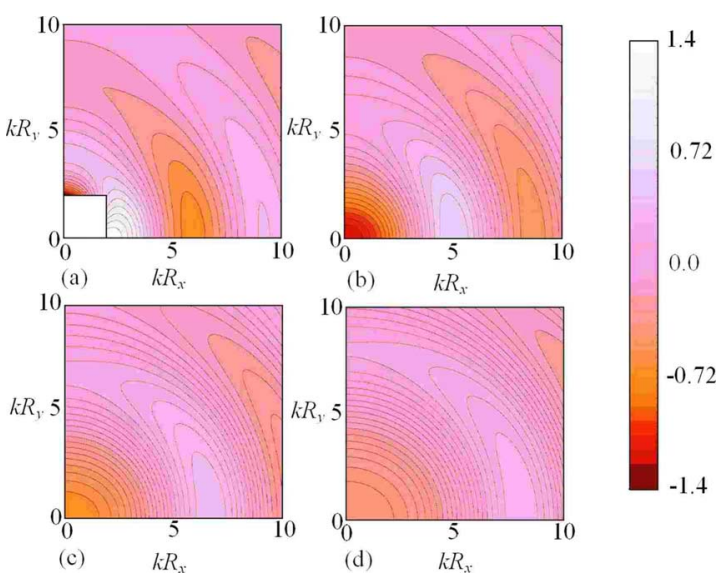

FIG. 1. (Color online) Contour maps of the optically induced interparticle interaction potential for two particles trapped in maxima of irradiance of the standing wave formed by counterpropagating optical plane waves of identical wavelength and irradiance against $k R_{x}=k\left(r_{x}^{(A)}-r_{x}^{(B)}\right)$ and $k R_{y}=k\left(r_{y}^{(A)}-r_{y}^{(B)}\right)$. In (a), particles are trapped in the same plane of maximum irradiance, and in (b)-(d) they are trapped in different maxima of irradiance separated by $\pi / k, 2 \pi / k$, and $3 \pi / k$, respectively. Energy units are $I \chi^{(A)} \chi^{(B)} k^{3} /(4 \pi c)$.

$$
\langle\Delta E\rangle=\left(\frac{2 I \varepsilon_{0} \chi^{(A)} \chi^{(B)}}{c}\right) V_{x x}\left(k,\left(\mathbf{r}^{(A)}-\mathbf{r}^{(B)}\right)\right) g,
$$

where $g=\cos \left[k\left(r_{z}^{(A)}-r_{z}^{(B)}\right)\right]$ when $\Delta k \neq 0$ and no interference is present, and $g=2 \cos k r_{z}^{(A)} \cos k r_{z}^{(B)}$ when $\Delta k=0$ and a standing wave is formed; the irradiance $I$ for any of the counterpropagating beams is given by $\alpha^{(+)} \bar{\alpha}^{(+)} \hbar c^{2} k / V$. Since the fields of the two counterpropagating beams are assumed identical or very similar, we have also set $\alpha^{(-)}=\alpha^{(+)}$. We now study the energy surfaces generated by this form of interaction. Figure 1 illustrates the result for particles trapped in the standing wave of counterpropagating plane waves (when $\Delta k=0$ ); in Fig. 1(a), the two particles are trapped by gradient forces in the same maximum of irradiance of the interference pattern; in Figs. 1(b)-1(d), the two particles are trapped in two different maxima separated by $\pi / k, 2 \pi / k$, and $3 \pi / k$, respectively. The minimum in the position $\left(R_{x}, R_{y}, R_{z}\right)$

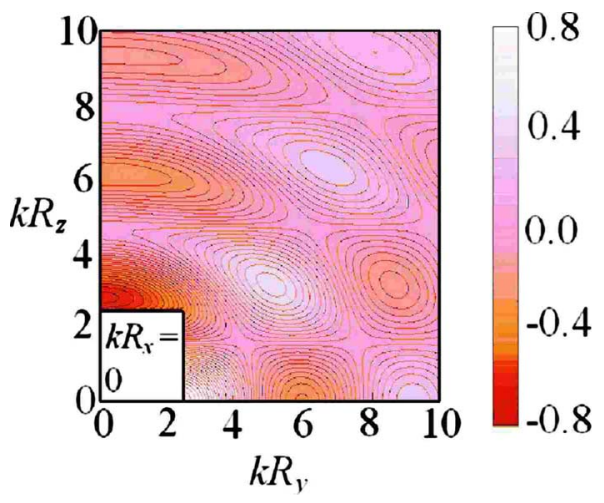

FIG. 2. (Color online) Optically induced interparticle energy surfaces on the $(x, y)$ plane, $\mathbf{R}=\left(R_{x}, R_{y}, R_{z}\right)$. The scale is in $I \chi^{(A)} \chi^{(B)} k^{3} /(4 \pi c)$ units.

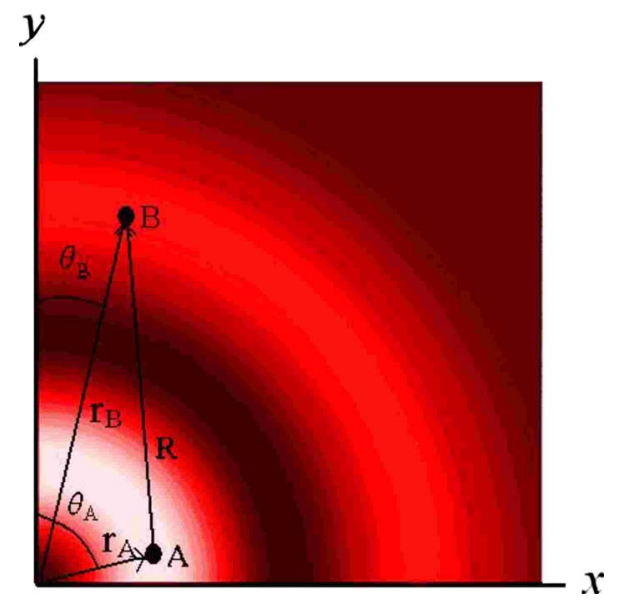

FIG. 3. (Color online) Transverse section of the waist of a Laguerre-Gaussian beam with two particles trapped in two different rings.

$=(6 / k, 0,0)$, which can be observed in Fig. 1(a), is responsible for transverse optical binding; the minima in the positions $(0,0, \pi / k),(0,0,2 \pi / k)$, and $(0,0,3 \pi / k)$ that can be observed in Figs. 2-4 are all contributions to the formations of chains of particles along the propagation direction of the field (longitudinal optical binding). Figure 2 shows a contour map of this optically induced interparticle potential energy in the case where $\Delta k \neq 0$, and consequently there is no interference. The contour map is drawn for a plane perpendicular to the polarization of the field, where multiparticle structures are expected to be located. As has been discussed in detail previously [37], this energy landscape can be responsible for

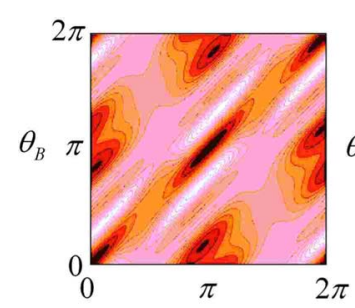

(a) $\quad \theta_{A}$

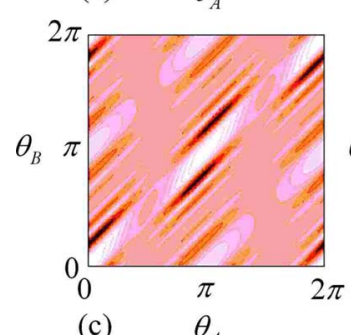

(c)

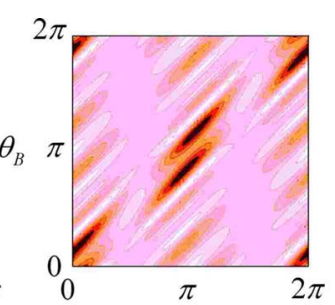

(b)

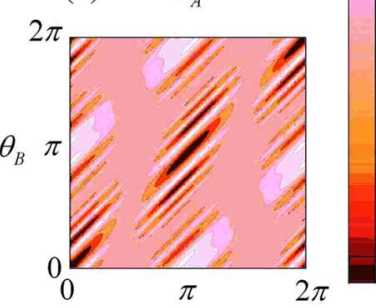

(d)

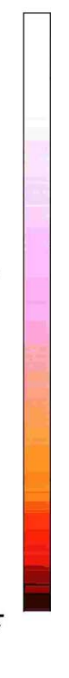

FIG. 4. (Color online) Contour map of the optically induced pair potential for two particles trapped in the two rings at the waist of a LG beam with $p=1, l=1$, and for $k \omega_{0}=$ (a) 10 , (b) 20 , (c) 30 , and (d) 40. The maximum of irradiance where the particle $A$ is present is at $r_{1}=0.47 k \omega_{0}$ from the beam center, and for the particle $B$ is at $r_{2}=1.51 k \omega_{0}$. Angles $\theta_{A}$ and $\theta_{B}$ are as shown in Fig. 1 . In the scale of the figure (a) black signifies $-1.06 \times 10^{-4}$, white $1.00 \times 10^{-4}$; (b) black $-2.43 \times 10^{-5}$, white $1.04 \times 10^{-5}$; (c) black $-6.22 \times 10^{-6}$, white $6.37 \times 10^{-6}$; (d) black $-2.47 \times 10^{-6}$, white $2.33 \times 10^{-6}$. Energy units are $\left(C_{2}^{1}\right)^{2} \chi_{0}^{(A)} \chi_{0}^{(B)} k^{5} /(4 \pi)$. 
the formation of longitudinal or transverse particle chains, and many other planar structures.

It is also worth considering the effect on this interaction of squeezing a coherent state. These states $\hat{S}(\zeta)|\alpha\rangle$ are generated by the operation of the squeeze operator, $\hat{S}(\zeta)$ $=\exp \left(\left\{\bar{\zeta} a^{(\lambda)}(\mathbf{k})^{2}-\zeta\left[a^{\dagger(\lambda)}(\mathbf{k})\right]^{2}\right\} / 2\right)$, on a coherent state $|\alpha\rangle$ $[34,35]$; here, $\zeta=s e^{i \varphi}$ is the complex squeeze parameter. The mean photon number in the resulting mode can be shown to be $\langle n\rangle=|\alpha|^{2}+\sinh ^{2} s$. Since the optically induced interparticle interaction is proportional to this number, the interaction increases sharply with the magnitude of the squeeze parameter $s$. In the following section we consider the optically induced interaction between particles trapped in Laguerre-Gaussian beams.

\section{INTERPARTICLE INTERACTION IN LAGUERRE- GAUSSIAN BEAMS}

Let us now consider the interaction between particles trapped in Laguerre-Gaussian (LG) beams. We denote this coherent state as $|\Lambda(\mathbf{k}, \lambda, l)\rangle$; the operations of the annihilation and creation operators on $|\Lambda(\mathbf{k}, \lambda, l)\rangle$ and $\langle\Lambda(\mathbf{k}, \lambda, l)|$, respectively, are as follows:

$$
a^{(\lambda)}(\mathbf{k})|\Lambda(\mathbf{k}, \lambda, l)\rangle=|\Lambda(r, z)| \exp [i \phi(r, z)]|\Lambda(\mathbf{k}, \lambda, l)\rangle,
$$

$$
\langle\Lambda(\mathbf{k}, \lambda, l)| a^{\dagger(\lambda)}(\mathbf{k})=\langle\Lambda(\mathbf{k}, \lambda, l)|\exp [-i \phi(r, z)]| \Lambda(r, z)|,
$$

where the eigenvalue in (15) is related to the positive propagation term of the classical field [36] and, from the complex amplitude of the field of a LG beam [38], we write $|\Lambda(r, z)|$ and $\phi(r, z)$ within the paraxial approximation as follows:

$$
\begin{gathered}
\left(\frac{\hbar c k \varepsilon_{0}}{2 V}\right)^{1 / 2}|\Lambda(z, r)|=\frac{C_{p}^{|l|}}{w(z)}\left(\frac{\sqrt{2} r}{w(z)}\right)^{|l|} \exp \left(\frac{-r^{2}}{w^{2}(z)}\right) L_{p}^{|l|}\left(\frac{2 r^{2}}{w^{2}(z)}\right), \\
\phi(z, r, \theta)=-\frac{k r^{2} z}{2\left(z^{2}+z_{R}^{2}\right)}-l \theta+(2 p+l+1) \arctan \left(\frac{z}{z_{R}}\right) .
\end{gathered}
$$

Here, $C_{p}^{|l|}$ is the normalization constant; $w(z)=w_{0} \sqrt{1+z^{2} / z_{R}^{2}}$ is the radius of the beam at $z$, where $w_{0}$ is the Gaussian beam waist at $z=0 ; L_{p}^{|l|}(x)$ is the generalized Laguerre polynomial of order $p$ and argument $x ;\left[(2 p+l+1) \arctan \left(z / z_{R}\right)\right]$ is known as the Gouy phase and $2 z_{R}$ is the Rayleigh range, a measure of $z$ over which collimation is sustained. The location of a particle $\xi$ in the beam is then expressed using the same parameters with $\xi$ as a subscript, $\left(z_{\xi}, r_{\xi}, \theta_{\xi}\right)$. Although a number state formulation for the interaction of particles with Laguerre-Gaussian beams has been developed [39], we adopt the one discussed above, since with this formulation the interaction of particles in different locations of the beam structure can be easily considered.

Let us now consider a system comprising two spherical particles $A$ and $B$ trapped by gradient forces in the waist of a

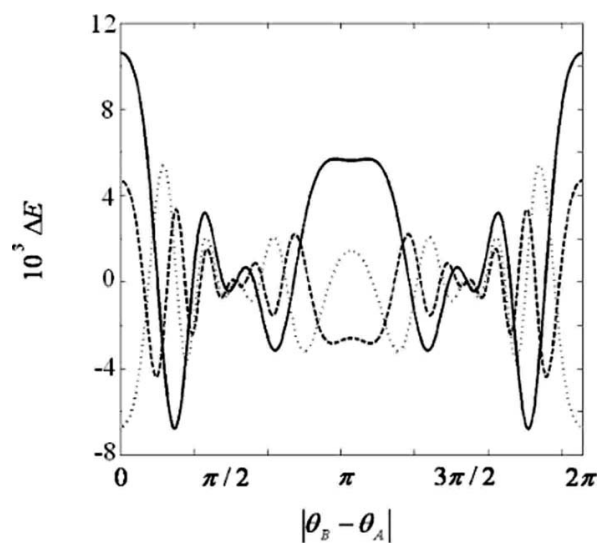

FIG. 5. Optically induced interparticle potential energy $\Delta E$ plotted against $\left|\theta_{B}-\theta_{A}\right|$ for two particles trapped in the two annuli of a LG beam with $p=1, l=1$, and $k \omega_{0}=20$ for the solid line, 30 for the dotted line, and 40 for the dashed line. Angles $\theta_{A}$ and $\theta_{B}$ are as shown in Fig. 3. The maxima of irradiance where the particles are present are as shown in Fig. 2. Energy units are $\left(C_{2}^{1}\right)^{2} \varepsilon \chi_{0}^{(A)} \chi_{0}^{(B)} k^{5} /(4 \pi)$. The graph equally applies to circularly and radically polarized beams.

beam of this type. We consider the case of particles located in different rings to extend previous results on optical binding in a single annulus [40]. Particle $A$ is in the first ring, the one of maximum irradiance and radius $r_{1}$, and particle $B$ is in the second ring $r_{2}$. The location of the particles can then be specified by the parameters $\left(z_{A}, r_{A}, \theta_{A}\right)=\left(0, r_{1}, \theta_{A}\right)$ and $\left(z_{B}, r_{B}, \theta_{B}\right)=\left(0, r_{2}, \theta_{B}\right)$, as is shown in Fig. 3 . The expectation value of the optically induced interparticle interaction potential may then be determined as in previous sections, and written as

$$
\begin{aligned}
\langle\Delta E\rangle= & \left(\frac{\hbar c k \varepsilon_{0}}{2 V}\right) \frac{\varepsilon_{0} \chi_{0}^{(A)} \chi_{0}^{(B)}\left|\Lambda\left(0, r_{1}\right)\right|\left|\Lambda\left(0, r_{2}\right)\right|}{4 \pi c R^{3}} \\
& \times \operatorname{Re}\left(\exp \left\{i\left[\phi\left(0, r_{2}, \theta_{B}\right)-\phi\left(0, r_{1}, \theta_{A}\right)\right]\right\}\right) \\
& \times\left(\sum _ { i = x , y } \left\{\left[1-3\left(R_{i} / R\right)^{2}\right](\cos k R+k R \sin k R)\right.\right. \\
& \left.\left.-\left[1-\left(R_{i} / R\right)^{2}\right] k^{2} R^{2} \cos k R\right\}\right)
\end{aligned}
$$

where $\chi_{0}^{(A)}$ and $\chi 0(B)$ are the susceptibilities of particles $A$ and $B$; the Cartesian components of the particle displacement vectors are given by $R_{x}=r_{2} \sin \theta_{B}-r_{1} \sin \theta_{A}$, $R_{y}=r_{2} \cos \theta_{B}-r_{1} \cos \theta_{A}$, and $R=\sqrt{R_{x}^{2}+R_{y}^{2}}$.

The spatial distribution of the potential energy (19), for a beam with a field defined by the LG parameters $p=1$ and $l$ $=1$, is illustrated in Figs. 4 and 5 for various field polarization conditions. The positioning of the potential energy maxima and minima determine the form of patterning in the optically configured potential energy surface. The energy minima, in particular, will determine the optimum locations for distributing optically trapped particles. The variation with $\theta_{A}$ and $\theta_{B}$ of features in the potential energy landscape, exhibited in Figs 4 and 5, is independent of $k$ and $w_{0}$ for any fixed value of $k w_{0}$. For example, increasing $k$ (equivalent to reducing the wavelength) by a factor of $n$, whilst also reduc- 
ing $w_{0}$ by the same factor, does not change the appearance of the landscape. However, the absolute energy values are a sensitive function of $k$ through an overall proportionality to $k^{5}$; small changes in this parameter can thus produce significant increases in the magnitude of the binding energiesnotice the units for the energy scales in these figures. For these figures we have chosen values of $k w_{0}$ that will satisfy the paraxial approximation. We now focus in more detail on the results.

Figure 4 shows contour maps of the interparticle interaction potential induced by a LG beam that is linearly polarized along the $y$ axis, for different values of $k w_{0}$. A general feature that may be observed is a pattern of four sequences of alternating minima and maxima, centred at $(\pi, \pi),(0,0)$, $(\pi, 0)$, and $(0, \pi)$. In these sequences, the number of maxima and minima increases with $k w_{0}$, in agreement with the linear increase of the ring perimeter with this parameter, and the oscillatory dependence of the interparticle potential on the relative displacement vector. Since the perimeter of the ring where particle $B$ is present is significantly larger than the one where $A$ resides, these sequences extended over a large range of $\theta_{B}$ and a small range of $\theta_{A}$.

Figure 5 shows the potential induced by a beam of the same wave-front topology as in Fig. 4, when the beam is either circularly or radially polarized; both polarizations lead to the same result, again exhibited for different values of $k w_{0}$. A number of potential energy minima and maxima of varying magnitudes, as a function of $\left|\theta_{B}-\theta_{A}\right|$, can be observed; the number of minima increases in approximately linear proportion to $k w_{0}$, and the individual features are in general distributed uniformly on $\left|\theta_{B}-\theta_{A}\right|$. These potential energy surfaces show that, by manipulating the parameter $k w_{0}$, the arrangement of particles on the individual rings of $\mathrm{LG}$ beams may be controlled.

\section{INTERPARTICLE INTERACTION INDUCED BY BROADBAND RADIATION}

In this section, we consider the optically induced potential induced by broadband light. It is well known that a broad white-light continuum can be generated from mode-locked laser light by means of supercontinuum generation [41]. The highly incoherent light resulting from this process has been used for the fabrication of optical traps with marked structural differences from those produced by monochromatic light [28]. Due to the small coherence length of the continuum radiation, such traps circumvent any problems due to interference between counterpropagating beams, or interference between the backscatter and beam fields [28,29], each of which may influence particle organization in studies using narrow bandwidth radiation. In these traps, the organization of particles along the Poynting vector proves attributable to optical binding - an optically induced form of particle coupling generally studied with monochromatic radiation [26].

For simplicity, the analysis addresses a single direction of beam throughput; an extension to counterpropagating beams is perfectly straightforward. The direction of polarization of the linearly polarized input is designated the $x$ axis, and the Poynting vector the $z$ axis. To begin, the induced electric polarization of a Rayleigh particle $\xi$ with electric susceptibility $\chi^{(\xi)}(k)=\chi^{(\xi)}(k,-k)$ for a mode with wave-vector modulus $k$ is written as [35]

$$
\begin{aligned}
P_{x}^{(\mathrm{ind}, \xi)}(k)= & -i\left(\frac{\hbar c k \varepsilon_{0}}{2 V}\right)^{1 / 2}\left[\chi^{(\xi)}(k) a(k) e^{i\left(k r_{z}^{(\xi)}-c k t\right)}\right. \\
& \left.-\chi^{(\xi)}(-k) a^{\dagger}(k) e^{-i\left(k r_{z}^{(\xi)}-c k t\right)}\right]
\end{aligned}
$$

where $V$ is an arbitrary quantization volume; the scalar $r_{z}^{(\xi)}$ is the projection on the $z$ axis of the position of the particle $\xi ; \varepsilon$ is the permittivity of the medium within which the particles are supported; $\chi^{(\xi)}(-k)=\chi^{(\xi)}(-k, k)$. The time average (av) of the energy shift operator for the optically induced interaction between particles $A$ and $B$ can be written using the QED method cast in terms of induced dipoles as follows [31]

$$
\Delta E_{\mathrm{av}}=\sum_{k}\left\{\operatorname{Re}\left[P_{x}^{(\mathrm{ind}, A)}(k) V_{x x}(k, \mathbf{R}) P_{x}^{(\mathrm{ind}, B)}(k)\right]\right\}_{\mathrm{av}},
$$

where $\mathbf{R}=\left(r_{x}^{(B)}-r_{x}^{(A)}, r_{y}^{(B)}-r_{y}^{(A)}, r_{z}^{(B)}-r_{z}^{(A)}\right)$ is the interparticle vector between the particles $A$ and $B$. Inserting Eq. (20) in (21), and using the commutation relation $a^{\dagger}(k) a(k)$ $-a(k) a^{\dagger}(k)=1$, the energy shift can be expressed as

$$
\begin{aligned}
\Delta E_{\mathrm{av}}= & \operatorname{Re}\left[\sum_{k}\left(\frac{\hbar c k a^{\dagger}(k) a(k)}{2 V}\right)\right. \\
& \times \varepsilon_{0}\left[\chi^{(A)}(k) \chi^{(B)}(-k) e^{i k\left(r_{z}^{(A)}-r_{z}^{(B)}\right)}\right. \\
& \left.\left.+\chi^{(A)}(-k) \chi^{(B)}(k) e^{i k\left(r_{z}^{(B)}-r_{z}^{(A)}\right)}\right]\right] V_{x x}(k, \mathbf{R}),
\end{aligned}
$$

where we have neglected the Casimir-Polder potential (which is relatively insignificant over the distances usually associated with optical binding), as shown in Sec. I. The discrete sum over $k$ is now replaced with the following continuum integral [35]:

$$
\sum_{k} a^{\dagger}(k) a(k) f(k) \rightarrow \int a^{\dagger}(\omega) a(\omega) f(\omega / c) d \omega,
$$

where $a(\omega)$ and $a^{\dagger}(\omega)$ denote the continuum photon annihilation and creation operators, respectively, at frequency $\omega$; $f(k)$ is an arbitrary function of $k$. The expectation value of the energy shift for the continuum state $|\{x\}\rangle$ is accordingly expressed as

$$
\begin{aligned}
\left\langle\Delta E_{\mathrm{av}}\right\rangle= & \operatorname{Re}\left[\frac{\varepsilon_{0}}{2 c} \int S(\omega) V_{x x}(\omega / c, \mathbf{R})\right. \\
& \times\left(\chi^{(A)}(\omega) \chi^{(B)}(-\omega) e^{i\left[\omega\left(r_{z}^{(A)}-r_{z}^{(B)}\right) / c\right]}\right. \\
& \left.\left.+\chi^{(A)}(-\omega) \chi^{(B)}(\omega) e^{-i\left[\omega\left(r_{z}^{(A)}-r_{z}^{(B)}\right) / c\right]}\right)\right] d \omega,
\end{aligned}
$$

where the spectral irradiance of the source is given by

$$
S(\omega)=\left(\frac{c \hbar \omega\left\langle\{x\}\left|a^{\dagger}(\omega) a(\omega)\right|\{x\}\right\rangle}{V}\right) .
$$

Equation (24) is applicable to optical states with any phase uncertainty and to multimode states, where the occupation number may change in space. In the following section it is 


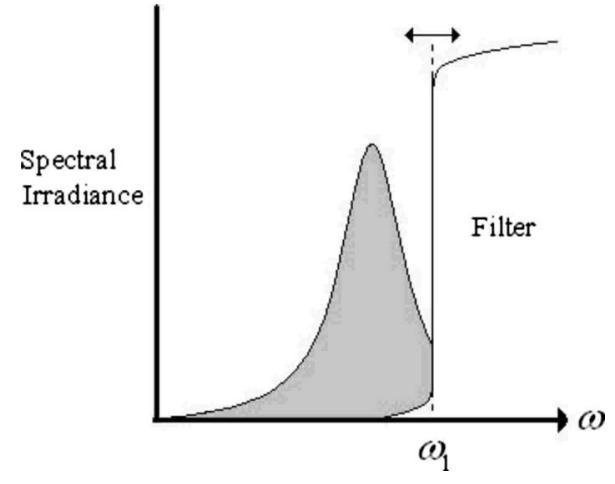

FIG. 6. Spectral irradiance of light, with Lorentzian spectrum, transmitted through a long-wavelength pass filter.

shown how the application of this result affords a method for the fabrication of moldable particle arrays using continuum light.

\section{Moldable particle arrays}

Recent research has shown that particle arrays whose formation is optically induced can be micromanipulated with precision by a secondary field [42]. Here, a method by means of which optically fabricated arrays can be molded using continuum light is described. For convenience the spectral irradiance of the light is assumed to have Lorentzian form:

$$
S(\omega)=\frac{K}{\left(\omega_{0}-\omega\right)^{2}+\gamma^{2}},
$$

where $\omega_{0}$ determines the center frequency; the parameter $K$ determines the maximum, and $\gamma$ the width of the function. For simplicity, each of the interacting pair of particles $A$ and $B$ is assumed to comprise centers (molecules) whose optical response is dominated by the ground state, $|0\rangle$, and one excited state, $|r\rangle$, and consequently the particle susceptibilities are given by

$$
\chi^{(A)}(\omega)=\chi^{(B)}(\omega)=\frac{2 N}{3 \hbar} \frac{\omega_{r 0}^{2}\left|\mu^{r 0}\right|^{2}}{\varepsilon_{0}\left(\omega_{r 0}^{2}-\omega^{2}\right)},
$$

where $N$ is the number of optical centers per particle; $\hbar \omega_{r 0}$ is the difference of energy between the states $|0\rangle$ and $|r\rangle ; \mu^{r 0}$ $=\langle r|\mu| 0\rangle$ is the transition moment associated with the electronic transition $|r\rangle \rightarrow|0\rangle$. To avoid absorption and any attendant thermal effects (which might change optical properties), and to observe the dependence of the interparticle potential energy surfaces on spectrally different forms of irradiance, part of the light can be filtered. Experimentally, this is expedited by the use of long-pass filters, whose effect on the spectral irradiance is illustrated in Fig. 6. Under these conditions, the interparticle potential energy for each particle pair can be written as follows:
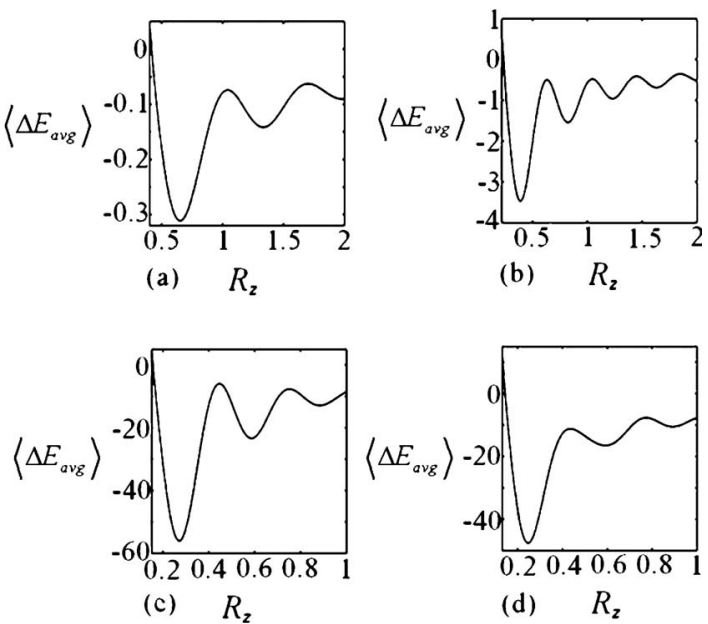

FIG. 7. Optically induced interparticle potential given by Eq. (28) for $\omega_{0}=10 \gamma, \omega_{r 0}=20 \gamma$, and $\omega_{1}=$ (a) $5 \gamma$, (b) $8 \gamma$, (c) $12 \gamma$, and (d) $15 \gamma$. The units of energy and distance are $400 K N^{2}\left|\mu^{r 0}\right|^{4} /\left(c \varepsilon_{o} \hbar^{2}\right)$ and $c / \gamma$, respectively. The Poynting vector is along the $z$ axis and the polarization is along the $x$ axis.

$$
\begin{aligned}
\left\langle\Delta E_{\mathrm{av}}\right\rangle= & \operatorname{Re}\left[\frac{4 K N^{2}\left|\mu^{r 0}\right|^{4} \omega_{r 0}^{2}}{9 c \hbar^{2} \varepsilon_{0}}\right. \\
& \times \int_{0}^{\omega_{1}} \frac{1}{\left(\omega_{r 0}^{2}-\omega^{2}\right)^{2}} \frac{V_{x x}(\omega / c, \mathbf{R})}{\left(\omega_{0}-\omega\right)^{2}+\gamma^{2}} \\
& \left.\times \cos \left(\frac{\omega\left(r_{z}^{(A)}-r_{z}^{(B)}\right)}{c}\right) d \omega\right] .
\end{aligned}
$$

The integral can then be evaluated numerically. It is now possible to explore the effect of choosing different spectral intervals by using pass filters with different wavelength characteristics (hence different values of $\omega_{1}$ ). The graphs in Fig. 7 show the optically induced interparticle potential along the Poynting vector of the beam for different values of $\omega_{1}$. Having regard to the ordinate axis values, this figure reveals that, by increasing the region of the spectrum interacting with the particles (i.e., by a positive shift in $\omega_{1}$ ), the magnitude of the energy shift is significantly increased in the first three graphs; this is a simple consequence of the corresponding increase in irradiance. More significantly, the sequences of minima within each such curve form a series in which each is separated from the next by about the same distance, a condition that favors the formation of particle chains [20]. With a change of $\omega_{1}$, the locations of all the minima in every graph are displaced in a positive or negative sense in identical proportion. This suggests that any variation in the position of the first minimum is indicative of a corresponding change in the length of particle chains along the Poynting vector. The graph in Fig. 8 illustrates the dependence of the location of the first potential energy minimum as a function of $\omega_{1}$, revealing that optically induced chains may be controllably extended or contracted by filtering appropriate frequency regions of the continuum input radiation.

\section{CONCLUSION}

A quantum electrodynamical analysis of the optically induced interparticle potential has been performed with a focus 


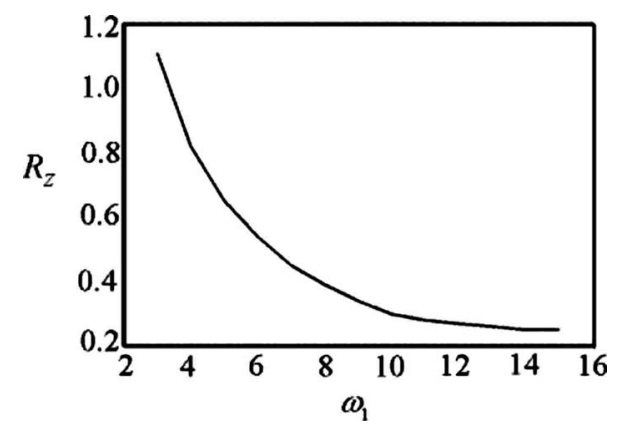

FIG. 8. Position of the first energy minimum of the potential interaction energy illustrated in Fig. 7 as a function of $\omega_{1}$, representing pass filters with different wavelength characteristics (see text, and Fig. 6). The units of distance and frequency are $c / \gamma$ and $\gamma$, respectively

on the differences produced by various types of optical field. Specifically, number states, coherent states, squeezed coherent states, Laguerre-Gaussian (twisted or vortex) beams, and broadband light have been analyzed. Among other results it has been shown, for example, that a variety of particle chains and other structures can be fabricated in counterpropagating beams; that the organization of particles in the rings of Laguerre-Gaussian beams can be directly controlled by varying the product of the wave number and the spot size at the beam waist; that the length of particle chains can be controlled by tailoring broadband light with wavelength cutoff filters. Such results exhibit the rich scope for optical fabrica- tion and management of diverse types of particle structures, solely using the properties of states of light. Further studies of optical manipulation with broadband light are the subject of ongoing research.

It is important to place the results in a proper context of their potential experimental realization. In principle, the lower the magnitudes of the optical property tensors and the masses of the optically trapped particles, the higher the probability that noise and thermal energy might diminish these interactions. In consequence, optical binding forces are indeed more easily observable for microparticles than for nanoparticles, and the phenomenon has a significantly lower probability for detection between molecules. However, observations of optical binding are generally performed using optically trapped species with an extremely low translational temperature, and the observed structures are remarkably stable - to the extent that forced perturbations can be used to ascertain restoring forces through a study of the oscillatory motions centered on local potential energy minima [15]. Given that there has been significant success when trapping single atoms in optical traps [43], there are good reasons to suppose that very small particles will display the interactions we have described, in suitably cooled systems.

\section{ACKNOWLEDGMENTS}

We gratefully acknowledge comments from Luciana C. Dávila Romero, David S. Bradshaw, and Jamie M. Leeder.
[1] E. A. Power and T. Thirunamachandran, Phys. Rev. A 51, 3660 (1995).

[2] P. W. Milonni, The Quantum Vacuum: An Introduction to Quantum Electrodynamics (Academic, San Diego, 1994).

[3] T. Thirunamachandran, Mol. Phys. 40, 393 (1980).

[4] M. M. Burns, J.-M. Fournier, and J. A. Golovchenko, Phys. Rev. Lett. 63, 1233 (1989).

[5] P. C. Chaumet and M. Nieto-Vesperinas, Phys. Rev. B 64, 035422 (2001).

[6] S. A. Tatarkova, A. E. Carruthers, and K. Dholakia, Phys. Rev. Lett. 89, 283901 (2002).

[7] J. Ng, Z. F. Lin, C. T. Chan, and P. Sheng, Phys. Rev. B 72, 085130 (2005).

[8] M. Guillon, O. Moine, and B. Stout, Phys. Rev. Lett. 96, 143902 (2006).

[9] N. K. Metzger, E. M. Wright, and K. Dholakia, New J. Phys. 8, 139 (2006).

[10] N. K. Metzger, E. M. Wright, W. Sibbett, and K. Dholakia, Opt. Express 14, 3677 (2006).

[11] T. Cizmar, V. Kollarova, Z. Bouchal, and P. Zemánek, New J. Phys. 8, 43 (2006).

[12] V. Karásek, K. Dholakia, and P. Zemanek, Appl. Phys. B: Lasers Opt. 84, 149 (2006).

[13] F. Chen, G. L. Klimchitskaya, V. M. Mostepanenko, and U. Mohideen, Opt. Express 15, 4823 (2007).

[14] J. Rodríguez, L. C. Dávila Romero, and D. L. Andrews, J.
Nanophotonics 1, 019503 (2007).

[15] N. K. Metzger, R. F. Marchington, M. Mazilu, R. L. Smith, K. Dholakia, and E. M. Wright, Phys. Rev. Lett. 98, 068102 (2007).

[16] R. G. Crisp and D. L. Andrews, Proc. SPIE 6483, 648304 (2007).

[17] S. Ahlawat, R. Dasgupta, and P. K. Gupta, Proc. SPIE 6535 , 65350W (2007).

[18] V. Karásek and P. Zemánek, J. Opt. A, Pure Appl. Opt. 9, S215 (2007).

[19] D. M. Gherardi, A. E. Carruthers, T. Cizmar, E. M. Wright, and K. Dholakia, Appl. Phys. Lett. 93, 041110 (2008).

[20] J. Rodríguez, Opt. Lett. 33, 2197 (2008).

[21] J. Rodríguez and D. L. Andrews, Opt. Lett. 33, 2464 (2008).

[22] J. Rodríguez, L. C. Dávila Romero, and D. L. Andrews, Proc. SPIE 6905, 69050H (2008).

[23] L. C. Dávila Romero, J. Rodríguez, and D. L. Andrews, Proc. SPIE 6988, 69880L (2008).

[24] L. C. Dávila Romero, J. Rodríguez and D. L. Andrews, Opt. Commun. 281, 865 (2008).

[25] L. C. Dávila Romero and D. L. Andrews, in Structured Light and its Applications, edited by D. L. Andrews (Academic, Burlington MA, 2008), pp. 79-105.

[26] M. Guillon and B. Stout, Phys. Rev. A 77, 023806 (2008).

[27] M. Kawano, J. T. Blakely, R. Gordon, and D. Sinton, Opt. Express 16, 9306 (2008). 
[28] M. Li and J. Arlt, Opt. Commun. 281, 135 (2008).

[29] R. F. Marchington, M. Mazilu, S. Kuriakose, V. GarcésChávez, P. J. Reece, T. F. Krauss, M. Gu, and K. Dholakia, Opt. Express 16, 3712 (2008).

[30] V. Karásek, T. Cizmár, O. Brzobohatý, P. Zemánek, V. GarcésChávez, and K. Dholakia, Phys. Rev. Lett. 101, 143601 (2008).

[31] A. Salam, Phys. Rev. A 73, 013406 (2006).

[32] D. P. Craig and T. Thirunamachandran, Molecular Quantum Electrodynamics: An Introduction to Radiation Molecule Interactions (Dover, Mineola, NY, 1998).

[33] M. A. Henesian and S. N. Dixit, Proc. SPIE 1870, 2 (1993).

[34] S. M. Barnett and P. M. Radmore, Methods in Theoretical Quantum Optics (Oxford University Press, Oxford, 1997).

[35] R. Loudon, The Quantum Theory of Light (Oxford University Press, New York, 2000).

[36] A. D. Bandrauk, Quantum and Semiclassical Electrodynamics:
Molecules in Laser Fields (Marcel Dekker, New York, 1994), pp. 1-70.

[37] J. Rodríguez, L. C. Dávila Romero, and D. L. Andrews, Phys. Rev. A 78, 043805 (2008).

[38] G. Nienhuis, Structured Light and Its Applications: An Introduction to Phase-Structured Beams and Nanoscale Optical Forces (Academic, Burlington MA, 2008), pp. 19-60.

[39] L. C. Dávila Romero, D. L. Andrews, and M. Babiker, J. Opt. B: Quantum Semiclassical Opt. 4, S66 (2002).

[40] D. S. Bradshaw and D. L. Andrews, Opt. Lett. 30, 3039 (2005).

[41] R. R. Alfano, The Supercontinuum Laser Source (Springer, New York, 1989).

[42] D. L. Andrews and J. Rodríguez, Opt. Lett. 33, 1830 (2008).

[43] D. Meschede and A. Rauschenbeutel, Adv. At., Mol., Opt. Phys. 53, 75 (2006). 